\title{
Water Resources Management Policy in Algeria: Strategies and Means
}

\author{
${ }^{1}$ Bousmaha Ahmed ${ }^{*}{ }^{2}$ Boulkaibet Aissa \\ ${ }^{1}$ Faculty of Earth Sciences and Architecture, University of Oum El Bouaghi, Algeria \\ ${ }^{2}$ Institute of Management of Urban Techniques, University of Oum El Bouaghi, Algeria
}

\begin{abstract}
In Algeria, the issue of water management is of great importance and represent a major contest in the context of a chronic shortage of rainfall from global warming. The catastrophic situation of water resources in Algeria during the years 2000 and 2001 was mainly due to drought that has prompted the government to give priority to this vital field. After a diagnosis of the situation of water resources, Algeria has adopted effective institutional, legal and technical arrangements for the sound management of water resources in order to overcome the inconsistencies that have profoundly influenced during antecedent decades. However, the expected results are not yet satisfactory and the state water resources remains negative. In this context, it is necessary to adopt new practices and efficient ways of management "sustainable" our water heritage in the context of a policy of good governance of water resources must go through a dialogue between the different actors and integrated management of all economic and all planning activities.
\end{abstract}

Keywords: Water Resources, Climate Change, Water Deficit, Integrated Management Of Water Resources, Water Security.

\section{Introduction}

L'accès durable aux ressources en eau est une préoccupation majeure qui concerne tous les pays du bassin méditerranéen. Le changement climatique et la croissance urbaine et démographique attendus dans la région, risquent d'aggraver la situation de stress hydrique qui frappe déjà la plupart des pays du Sud et de l'Est de la Méditerranée [1]. En effet, le 4ème rapport du Groupe d'experts intergouvernemental sur l'évolution du climat (GIEC) est sans équivoque : le bassin méditerranéen figurera au cours du 21ème siècle au rang des régions les plus sévèrement touchées par les changements climatiques. Les données climatiques relevées dans les pays du pourtour de la Méditerranée indiquent un réchauffement durant ce siècle estimé à plus de $1^{\circ} \mathrm{C}$ avec une tendance accentuée durant les 30 dernières années [2]. La zone méditerranéenne est l'une des zones du monde les plus vulnérables au changement climatique [3]. « Dans son quatrième rapport paru en 2007, le Groupe d'expert Intergouvernemental sur l'Evolution du Climat (GIEC) combine vingt-cinq modèles climatiques globaux afin d'évaluer les impacts du changement climatique aux horizons 2050 et 2100. Selon leurs travaux, une hausse des températures de +2 à $+3^{\circ} \mathrm{C}$ est à prévoir en région méditerranéenne à $1^{\prime}$ horizon 2050 , puis de +3 à $5^{\circ} \mathrm{C}$ à 1 'horizon $2100 »$ [4].

Les pays méditerranéens apparaissent donc particulièrement vulnérables aux conséquences du changement climatique sur les ressources en eau et leurs usages, en raison notamment d'une dégradation croissante de ces ressources (surexploitation, pollution, salinisation, diminution de la pluviométrie) et d'une augmentation des demandes dans les secteurs agricole, urbain et énergétique [5]. De manière générale, l'ensemble des pays méditerranéens pourrait souffrir de pénuries du fait du changement climatique, qui affecte les quantités d'eau disponibles, et de l'augmentation de la demande en eau [6]. Cette situation implique la mise en place de mesures adaptatives et par la mise en place d'une politique de l'eau axée sur la rareté croissante de cette ressource.

En Algérie, les conditions climatiques qui prévalent depuis trois décennies ont une influence négative sur la ressource en eau [7]. Dans ce contexte, les changements climatiques sont considérés en Algérie comme un facteur clé dans la précarité des ressources en eau. Ils sont devenus un thème prioritaire dans la stratégie 
nationale de l'aménagement du territoire et de son développement durable. L'Algérie a été particulièrement vulnérable aux changements climatiques et au réchauffement de la planète qui a marqué le 20ème siècle [8].

L'Algérie ainsi que plusieurs pays méditerranéens se trouvent au-dessous du seuil moyen de $1000 \mathrm{~m} 3 / \mathrm{an}$ et par habitant, situation dans laquelle des tensions apparaissent entre les besoins et les ressources. Les ressources tombent sous le "seuil de pénurie" de $500 \mathrm{~m} 3 /$ an de ressource par habitant [9]. Les besoins des activités économiques, la croissance démographique et surtout «les effets du réchauffement climatique ne feront que renforcer les tendances actuelles eau » [10]. Le déficit en eau, qui sera amplifié par le phénomène de changement climatique, nécessite la mise en place d'une gestion rationnelle de cette ressource.

La situation catastrophique en matière des ressources en eau qu'a connue l'Algérie durant les années 2000 et 2001, due principalement à la sècheresse a incité les pouvoirs publics d'accorder une priorité à ce secteur vital.

La nouvelle politique de l'eau avait commencé au début des années 2000 afin de réaliser ces objectifs suivants [11] :

- Accroître et sécuriser la mobilisation de ressources en eau conventionnelles (renouvelables et fossiles) et non conventionnelles (dessalement et eaux usées épurées)

- Améliorer l'accès à l'eau à travers la réhabilitation et l'extension des systèmes d'alimentation en eau potable et le renforcement de la qualité de service.

- Améliorer l'accès à l'assainissement et protéger les écosystèmes hydriques au moyen de la réhabilitation et l'extension des systèmes d'assainissement et la réalisation de nouvelles stations d'épuration.

- Soutenir la stratégie de sécurité alimentaire avec l'extension des zones irriguées.

Selon le Ministère des Ressources en Eau (2012), les ressources en eau en Algérie sont estimées à environ 17 milliards de $\mathrm{m}^{3}$, pour $600 \mathrm{~m}^{3}$ annuellement mobilisables par habitant, soit un volume nettement en dessous des normes internationales admises (1000 m3 par habitant et par an). Cette ration fait de l'Algérie un pays situé sous le seuil de rareté, traduisant un déficit en eau. Outre la rareté de la ressource, une grande disparité spatiale en termes de répartition de la ressource et une insuffisance dans la gestion de l'eau. Cette ressource vitale est menacée dans sa qualité et dans sa quantité. Le dessalement des eaux de mer reste une solution sûre, vu la sécheresse qui sévit ces dernières années en Algérie [12]. Malgré la construction de nouveaux barrages et le recours au dessalement, l'Algérie enregistrera un déficit en eau de 1 milliard de $\mathrm{m}^{3}$ d'ici l'an 2025 dans le cas d'une mauvaise gestion de l'eau et d'une non maitrise des ressources non conventionnelles [13].

Le changement climatique peut accentuer les phénomènes climatiques extrêmes comme les sécheresses chroniques notamment dans les Hauts Plateaux et la Steppe et peut ainsi constituer un facteur aggravant de la dégradation des ressources en eau de ces milieux sensibles (il s'agit des milieux arides et semi arides qui souffrent principalement de la désertification et de la sécheresse).

La problématique de l'eau en Algérie depuis plusieurs décennies montre que bientôt la demande sera supérieure aux ressources [14].

Les « zones naturelles » et « étages bioclimatiques » qui caractérisent l'espace algérien nous permettent d'appréhender son hétérogénéité. Une grande partie du territoire algérien est située sur les marges de l'aridité et, pour sa partie saharienne, dans le domaine aride. Ceci fait peser sur ces territoires une menace constante et les récentes années de sécheresse, 2000 et 2001, ont attiré l'attention sur la fragilité des ressources en eau en Algérie. Les besoins en eau ont longtemps été ressentis essentiellement pour l'utilisation agricole (irrigation) mais la forte croissance démographique et l'urbanisation, les nouvelles implantations industrielles, les équipements touristiques relèvent d'autres consommations en forte croissance. Dans ce contexte, une véritable « économie de l'eau » devra être mise en place pour éviter de trop grandes distorsions entre secteurs d'utilisation mais aussi entre régions inégalement dotées. 


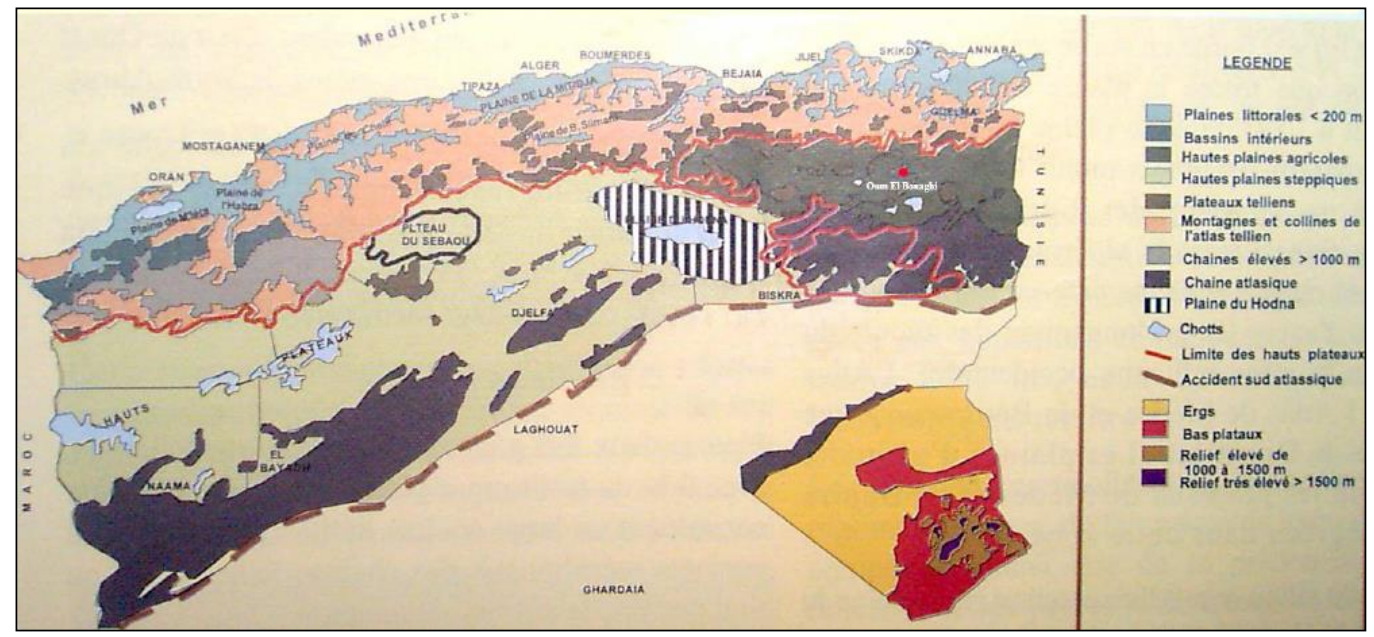

Fig. 1: Les zones naturelles en Algérie

\section{Source : Agence National d'Aménagement du Territoire (ANAT)}

La question de déficit hydrique, de demandes et de besoins des populations et de changement climatique, nous amènent à poser des questionnements quant à l'évolution conjointe de la pression sur la ressource en eau et de sa disponibilité dans les années à venir. Cependant, quel que soit le type d'aménagement hydraulique (petite, moyenne ou grande hydraulique pour capter les écoulements superficiels, forages profonds, pompage des nappes alluviales, puits artésiens pour mobiliser les eaux souterraines), l'irrégularité de l'alimentation en eau demeure en Algérie une contrainte fondamentale.

Dans ces conditions, quelle stratégie devons-nous mettre en place pour faire face aux défis du changement climatique ? Quelles solutions pour la problématique de l'eau en Algérie et comment réduire les insuffisances déjà perceptibles?

\section{Contrastes De L'espace Algerien}

\section{La Contrastes physiques}

L'espace algérien se caractérise par son hétérogénéité. Au nord, l'Atlas tellien forme avec l'Atlas saharien, plus au sud, deux ensembles de relief parallèles se rapprochant en allant vers l'est, et entre lesquels s'intercalent de vastes plaines et hauts plateaux. Les deux Atlas tendent à se confondre dans l'est de l'Algérie. Vers l'intérieur de l'est algérien les vastes chaines montagneuses des (Aurès) et de la Nememcha occupent la totalité de l'est algérien et elles sont délimitées par la frontière tunisienne. La bande du Tell, large de $80 \mathrm{~km}$ à 190 km, s'étend sur près de $1200 \mathrm{~km}$ de côte méditerranéenne. Elle est formée de chaînes de montagnes (l'Ouarsenis, le Chenoua, le Djurdjura, les Babors et les Bibans...) longeant le littoral et souvent séparées par des vallées, riches par leur flore et leur faune, abritant des cours d'eau comme la vallée du Chélif ou la vallée de la Soummam. Le mont Lalla-Khadîdja, en Kabylie où les montagnes sont recouvertes de neige en hiver, en est le point culminant et s'élève à 2308 mètres d'altitude. Les plaines du Tell abritent avec les vallées adjacentes la grande majorité des terres fertiles du pays [15].

Trois ensembles physiques caractérisent le territoire algérien :

- le littoral et l'ensemble Tellien occupent $4 \%$ de la superficie totale, 2.5 millions d'ha terres agricoles, riches en ressources et très menacées par la concentration excessive, ainsi que par l'urbanisation galopante (phénomène de littoralisation). Ces terres sont fragiles et peu résistantes à l'érosion.

- Les hauts plateaux occupent environ $9 \%$ de la superficie totale, 5 millions d'ha de terres agricoles. Le processus de désertification est important du fait de la sécheresse, de la fragilisation des sols soumis à l'érosion éolienne, de la faiblesse des ressources hydriques et de la pratique intensive de l'agropastoralisme [16].

- Le Sahara, ensemble désertique aride, couvre $87 \%$ du territoire algérien, les terres y sont pauvres, les conditions climatiques extrêmes. 
La présence de la chaîne montagneuse directement flanquée sur le littoral, interposée entre mer et espace intérieur arrête les précipitations venues du Nord ou du Nord-Ouest, localise les grands châteaux d'eaux du pays, assèche et continentalise l'intérieur. Elle organise l'hydrographie en une série de cours d'eaux parallèles et courts qui se précipitent vers la Méditerranée sans avoir pu se concentrer.

\section{Contrastes climatiques}

L'espace algérien se caractérise par des contrastes climatiques. Le nord du pays se caractérise par un climat méditerranéen (étés chauds et secs et hivers doux et pluvieux) et le sud saharien un climat désertique depuis le climat humide au Nord jusqu'au climat aride au Sud pour les étages bioclimatiques, et depuis le froid jusqu'au chaud pour les variantes thermiques.

- Dans le Tell et sur le littoral, les précipitations annuelles varient entre 400 et $1800 \mathrm{~mm}$ (tableau 1). Le massif du Djurdjura, situé en Kabylie et le massif de l'Edough, situé plus à l'est, sont les zones les plus arrosées de l'Algérie. En été les précipitations sont extrêmement rares et les chaleurs très fortes.

- Les hauts plateaux sont caractérisés par un climat semi-aride (pluviométrie comprise entre 250 et 400 $\mathrm{mm} / \mathrm{an}$ ). Les terres y ont une forte teneur en sel. Le processus de désertification est important du fait de la sécheresse, de la fragilisation des sols soumis à l'érosion éolienne, de la faiblesse des ressources hydriques et de la pratique intensive de l'agropastoralisme

- Le Sahara, ensemble désertique aride (pluviométrie moyenne entre 20 et $150 \mathrm{~mm} / \mathrm{an}$ ), les conditions climatiques sont extrêmes et les amplitudes thermiques sont très fortes.

\section{Tableau 1: Répartition des précipitations selon les régions géographiques du pays}

\begin{tabular}{|l|l|l|l|}
\hline Région & Ouest & Centre & Est \\
\hline Littoral & $400 \mathrm{~mm}$ & $700 \mathrm{~mm}$ & $900 \mathrm{~mm}$ \\
\hline Atlas Tellien & $600 \mathrm{~mm}$ & $700-1000 \mathrm{~mm}$ & $800-1400 \mathrm{~mm}$ \\
\hline Hauts Plateaux & $250 \mathrm{~mm}$ & $250 \mathrm{~mm}$ & $400 \mathrm{~mm}$ \\
\hline Atlas Saharien & $150 \mathrm{~mm}$ & $200 \mathrm{~mm}$ & $300-400 \mathrm{~mm}$ \\
\hline Sahara & $20-150 \mathrm{~mm}$ & $20-150 \mathrm{~mm}$ & $20-150 \mathrm{~mm}$ \\
\hline
\end{tabular}

Source : Agence Nationale de la Météorologie, 2009.

Les précipitations moyennes s'élèvent à $89 \mathrm{~mm} / \mathrm{an}$ sur l'ensemble du territoire algérien. La précipitation moyenne en Algérie représente 12,4 milliard m3 concentrée sur le littoral et la zone tellienne (fig. 3). Les températures sont variables selon les régions du pays. Au Sud, le thermomètre indique des variables entre 40 ${ }^{\circ} \mathrm{C}$ le jour et $5{ }^{\circ} \mathrm{C}$ la nuit. Par contre, le Nord a un climat méditerranéen. En été, les températures sont élevées. Les températures moyennes estivales et hivernales se situent entre $25^{\circ} \mathrm{C}$ et $11^{\circ} \mathrm{C}$ (fig. 4 ).

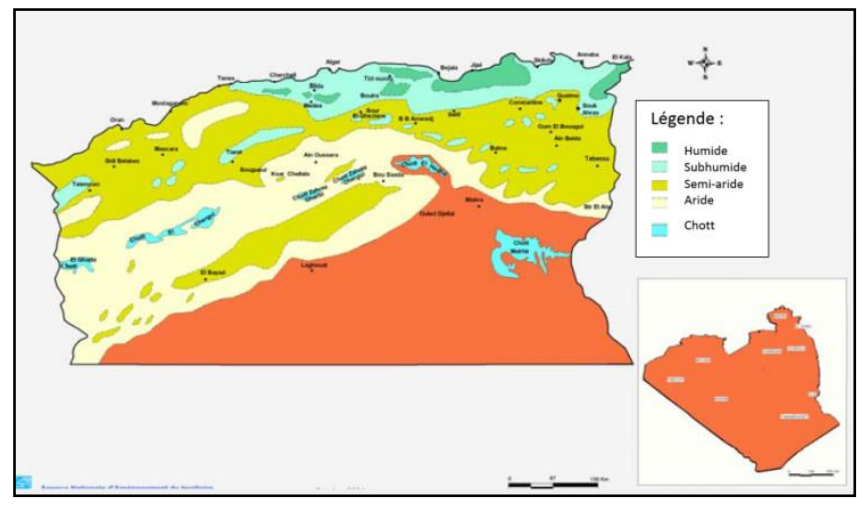

Fig. 2 : Etages bioclimatiques en Algérie

dans l'Algérie

Source : Agence Nationale de l'Aménagement du Territoire

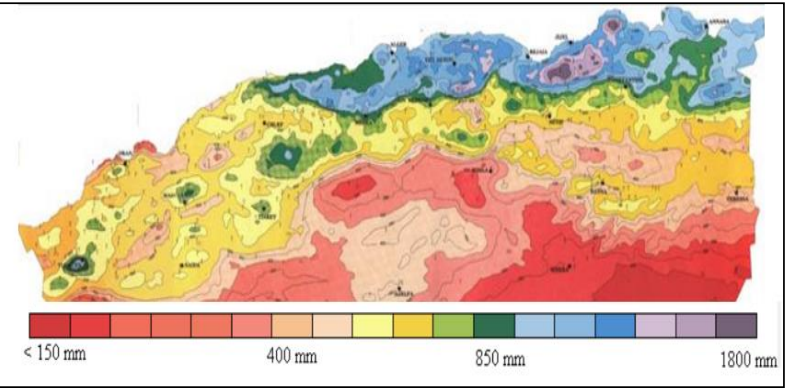

Fig. 3 : Précipitations (moyenne annuelle) du Nord en 2008

Source : Agence Nationale de

Ressources en Eau, 2008 


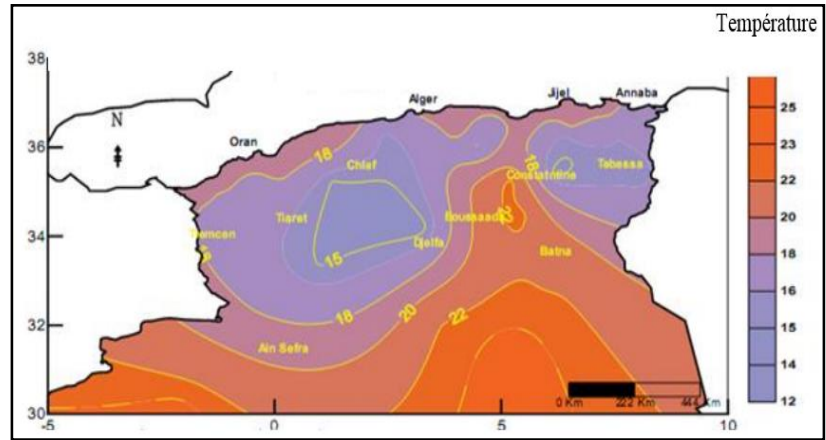

Figure 4 : Les températures moyennes en Algérie du Nord de 1950 à 2005

Source : Agence Nationale de la Météorologie, 2009.

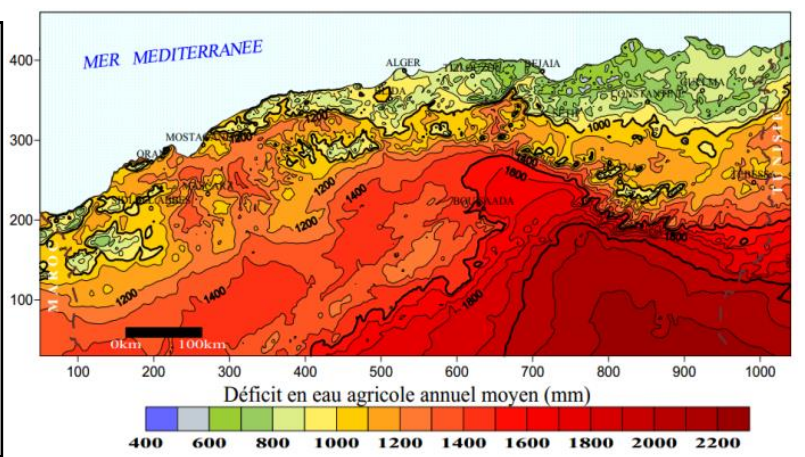

Fig. 5 : Zones de déficit hydrique moyen annuel

Les pluies sont très irrégulières et mal reparties à travers le pays et n'offrent donc qu'un très faible taux d'utilisation. Du littoral au Piémont saharien, la saison agronomique sèche s'étale sur 4 à 12 mois. On admet que la consommation globale d'un hectare de culture irriguée est de $5000 \mathrm{~m} 3 /$ an dans le Tell, de 8000 en zone steppique et de 10 à 20000 au Sahara [18].

Telle qu'illustré par la figure ${ }^{\circ} 5$, représentant les zones de déficit hydrique moyen annuel de l'Algérie du Nord, la répartition des déficits traduit la brutalité des contrastes climatiques en Algérie. Le stress hydrique est plus accusé dans la région de l'Ouest algérien. Les menaces du réchauffement climatique induiront des modifications du cycle de l'eau, une dégradation des terres agricoles et une baisse de la fertilité des sols.

Les caractéristiques climatiques en Algérie se résument aux précipitations qui sont irrégulières aux plans temporel et spatial et à un cycle long de sécheresse. Ce qui traduit l'accentuation de l'aridité avec une baisse de la pluviométrie se traduisant par une réduction du renouvellement des nappes aquifères et une diminution des apports au niveau des barrages [19] comme le montre bien l'exemple du barrage de BENI BAHDEL.

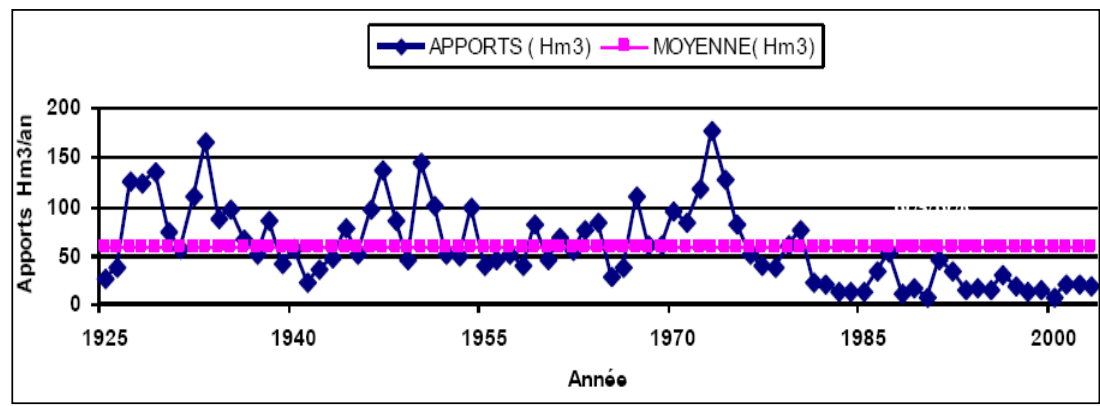

Fig. 6 : Diminution des apports annuels des barrages: Exemple de BENI BAHDEL

Source: Ministère des ressources en eau (2011)

Les évolutions socio-économiques actuelles ne sont guère rassurantes. Les terres arables diminuent au profit d'une urbanisation non maîtrisée. La croissance démographique, l'évolution des modes de vie etc., accroissent considérablement la demande en eau, quels que soient ses usages, réduisant la part allouée à l'agriculture. Les ressources en eau sont à l'évidence fortement conditionnées par les variables climatiques qui en déterminent les apports et la dynamique du cycle de l'eau (précipitations, températures). Consécutivement à l'augmentation de la température, l'évaporation plus importante des masses d'eau diminuerait les quantités de ressources disponibles.

\section{Changement climatique}

Les changements climatiques enregistrés durant les trois dernières décennies ont induit la réduction de la pluviométrie annuelle de plus de $20 \%$ au niveau de la steppe ainsi que l'intensification des cycles de sécheresses. Ceci a eu comme impacts l'amorcé d'un processus de dégradation des écosystèmes [20].

Dans son quatrième rapport paru en 2007, le Groupe d'expert Intergouvernemental sur 1'Evolution du Climat (GIEC) combine vingt-cinq modèles climatiques globaux afin d'évaluer les impacts du changement climatique aux horizons 2050 et 2100 . Selon leurs travaux et comme le montre la Figure $n^{\circ} 7$, une hausse des 
températures de +2 à $+3^{\circ} \mathrm{C}$ est à prévoir en région méditerranéenne à $1^{\prime}$ horizon 2050 , puis de +3 à $5^{\circ} \mathrm{C}$ à 1'horizon 2100 [21]. " Cette hausse des températures devrait réduire l'humidité relative de l'air et accroître la charge capacitive d'humidité de l'atmosphère. Le taux de saturation de l'air sera alors plus important entraînant une diminution de la nébulosité et donc des précipitations. Les évènements précipitants seront moins fréquents mais plus intenses, tandis que les périodes de sécheresse seront plus fréquentes et plus longues. La distribution spatiale et temporelle des précipitations s'en verra donc modifiée » [22].

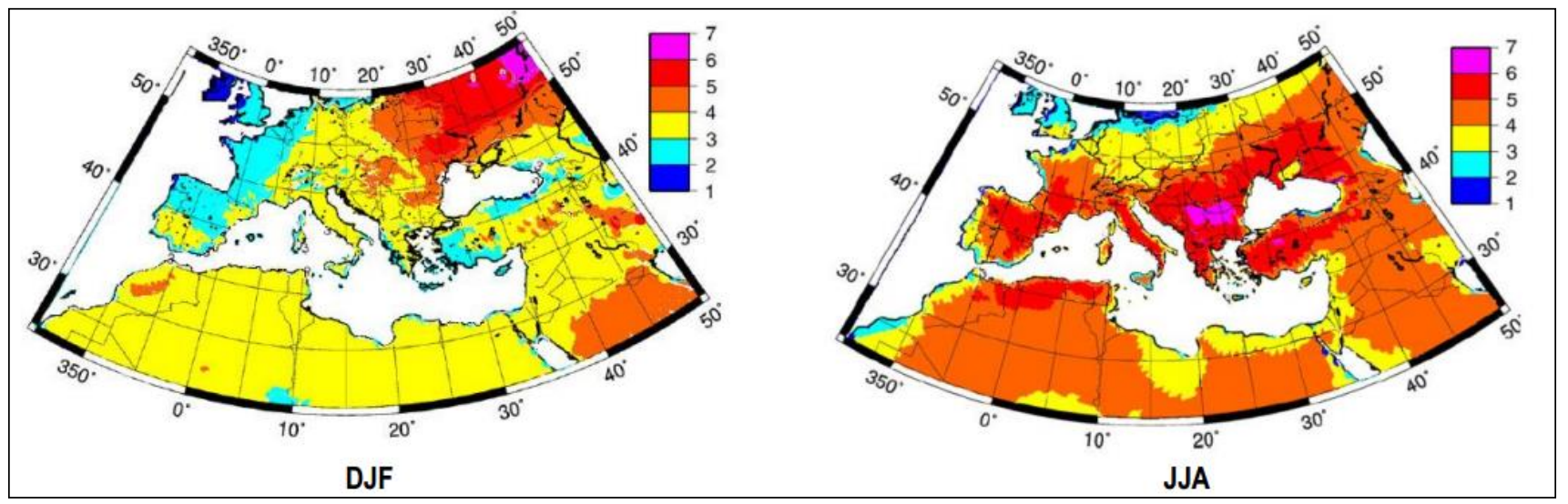

Fig. 7: Variations saisonnières des températures à l'Horizon 2100 par rapport à la période 1961-1990

Source : D'après SOMOT et al. 2008 [22].

Dans le contexte algérien, le changement climatique va renforcer l'aridité du climat et menacer gravement les milieux sensibles (hauts plateaux et zone steppique. La sécheresse intense et persistante observée durant les 20 dernières années, caractérisée par un déficit pluviométrique évalué à $10 \%$, a eu un impact négatif sur les régimes d'écoulement des cours d'eau, le niveau de remplissage des réservoirs de barrages et l'alimentation des nappes souterraines, entraînant des conséquences graves sur l'ensemble des activités socio-économiques du pays. Les premières estimations quantitatives, encore très approximatives, situent la réduction des débits d'oueds à une valeur moyenne de $15 \%$ à l'horizon 2030 . Le volume total des ressources superficielles mobilisables (par les barrages) estimé à 6,4 milliards de $\mathrm{m} 3$ /an pourrait se trouver réduit à une valeur maximale de 5,5 milliards de m3/an [23].

Dans ce contexte, l'impact des changements climatiques sera considérable sur les régimes d'écoulement des cours d'eau, niveau de remplissage des réservoirs de barrages, et sur l'alimentation des nappes souterraines, entraînant des conséquences sur l'ensemble des activités socio-économiques du pays. Les stratégies actuelles de développement prennent désormais en compte ces changements et envisagent certaines mesures d'adaptation.

Pour anticiper les impacts prévisibles du changement climatique, l'Algérie prévoit la mise en œuvre d'un plan d'action intégrant la réévaluation des ressources en eau renouvelables, l'adaptation des programmes de gestion des retenues actuelles et de construction de nouveaux barrages, la ré-estimation des capacités d'exploitation des eaux souterraines, les économies d'eau et le développement de ressources en eau non conventionnelles, ainsi que l'établissement de plans de gestion des risques [24].

\section{Le Diagnostic Des Ressources Hydriques En Algérie}

L'eau en Algérie est une ressource rare et limitée par les conditions climatiques, l'inégale distribution des précipitations, une ressource partagée et une forte pression démographique. Les potentialités hydriques en Algérie comprennent les eaux superficielles et les eaux souterraines qui sont réparties inégalement sur l'espace géographique dont la variabilité régionale est plus marquée à cause de la grande superficie de l'espace algérien qui se caractérise par sa diversité physique, bioclimatique, etc. Mais, dans sa majorité, l'Algérie est un pays aride et semi-aride.

En 2012, selon les estimations du Ministère des Ressources en Eau, l'Algérie totalise environ 17 milliard m3 en ressources en eau dont les eaux de surface représentent 9.8 milliard $\mathrm{m} 3$ et les eaux souterraines 7.1 milliard $\mathrm{m} 3$ (Tableau 2). L'apport principal en eau provient des eaux superficielles. Mais les pluies sont très irrégulières et mal reparties à travers le pays et n'offrent donc qu'un très faible taux d'utilisation.

Tableau 2: Répartition des ressources en eau selon les cinq régions hydrographiques du pays 


\begin{tabular}{|l|l|l|l|}
\hline Régions hydrographiques & Eaux de surface & Eaux souterraines & $\begin{array}{l}\text { Total de la } \\
\text { ressource }\end{array}$ \\
\hline Oranie - Chott Chergui & 1.0 milliard de $\mathrm{m}^{3}$ & 0.60 milliard de $\mathrm{m}^{3}$ & $\begin{array}{l}1.60 \text { milliard de } \\
\mathrm{m}^{3}\end{array}$ \\
\hline Chéliff - Zahrez & 1.5 milliard de $\mathrm{m}^{3}$ & 0.33 milliard $\mathrm{de}^{3}$ & $\begin{array}{l}1.83 \text { milliard de } \\
\mathrm{m}^{3}\end{array}$ \\
\hline Algérois -Hodna et Soummam & 3.4 milliard de $\mathrm{m}^{3}$ & 0.74 milliard de $\mathrm{m}^{3}$ & $\begin{array}{l}4.14 \text { milliard de } \\
\mathrm{m}^{3}\end{array}$ \\
\hline $\begin{array}{l}\text { Constantinois-Seybousse- } \\
\text { Mellegue }\end{array}$ & 3.7 milliard de $\mathrm{m}^{3}$ & 0.43 milliard de $\mathrm{m}^{3}$ & $\begin{array}{l}4.13 \text { milliard de } \\
\mathrm{m}^{3}\end{array}$ \\
\hline Sahara (la nappe Albienne) & 0.2 milliard de $\mathrm{m}^{3}$ & 5.00 milliard $\mathrm{de}^{3}$ & $\begin{array}{l}5.20 \text { milliard de } \\
\mathrm{m}^{3}\end{array}$ \\
\hline Total & 9.8 milliard de $\mathrm{m}^{3}$ & 7.10 milliard $\mathrm{de}^{3}$ & $\begin{array}{l}16.9 \text { milliard de } \\
\mathrm{m}^{3}\end{array}$ \\
\hline
\end{tabular}

Source : Ministère des Ressources en Eau, 2012.

\section{L'analyse SWOT}

SWOT est un acronyme signifiant Strengths, Weaknesses, Opportunities, Threats. En Français, AFOM qui signifie : Atouts, Faiblesses, Opportunités, Menaces.

Cet outil combine l'étude des forces et des faiblesses d'une organisation, d'un territoire, d'un secteur, etc. avec celle des opportunités et des menaces de son environnement, afin d'aider à la définition d'une stratégie de développement. Le but de cette analyse est de prendre en compte dans la stratégie, à la fois les facteurs internes et externes, en maximisant les potentiels des forces et des opportunités et en minimisant les effets des faiblesses et des menaces.

Tel qu'illustré par la matrice des ressources en eau en Algérie, les contraintes et les menaces qui se résument par la surexploitation des ressources souterraines dans le Nord du pays qui présentent des signes d'épuisement. La stratégie doit dessiner des alternatives et dépasser les menaces et parvenir à déceler les solutions qui soient en mesure d'atténuer les faiblesses et risques. Les contraintes évoquées dans l'analyse SWOT et les menaces signalées précédemment doivent être considérées.

En effet, près de $90 \%$ sont exploitées dans le nord du pays. De plus, la gestion de l'eau souffre de plusieurs contraintes dues principalement à l'inadaptation des systèmes de gestion, à l'envasement des barrages et aux pertes énormes à cause de la vétusté des réseaux.

Quant à l'entrée en exploitation de nouveaux barrages, elle va permettre d'atteindre une capacité de mobilisation de 10 milliards de $\mathrm{m} 3$ par an, permettant ainsi de réduire le volume annuel de déperdition de plus de 3 milliards de $\mathrm{m} 3$ [25]. L'achèvement et la mise en exploitation des nouvelles stations d'épuration d'eaux usées vont permettre d'affecter plus de 650 millions de m3 d'eaux épurées et traitées au secteur agricole. En plus, la littorale algérienne de $1200 \mathrm{~km}$ laisse entrevoir d'énormes possibilités en matière de désalinisation de l'eau de mer qui pourrait être une solution d'avenir.

Les ressources hydriques actuelles sont par ailleurs fragilisées par une forte démographie et d'importants besoins saisonniers touristiques et agricoles. Ces pressions sur les ressources en eau s'accompagnent d'une dégradation croissante de leur qualité et de leur surexploitation.

Tableau 4 : Matrice ressources en eau en Algérie 


\begin{tabular}{|c|c|}
\hline $\begin{array}{l}\text { - Des ressources souterraines importantes et } \\
\text { de qualité encore préservée. } \\
\text { - De grandes étendues recueillant un volume } \\
\text { ruisselé non négligeable, malgré la faible } \\
\text { pluviométrie. } \\
\text { - Importance des espaces à vocation } \\
\text { pastorale, moins consommateurs d'eau que } \\
\text { l'agriculture }\end{array}$ & $\begin{array}{l}\text { - Faiblesse et irrégularité de la pluviométrie. } \\
\text { - Contexte favorable à l'érosion et à l'envasement des } \\
\text { barrages. } \\
\text { - Faible connaissance du potentiel valorisable en eaux } \\
\text { souterraines. } \\
\text { - Absence de contrôle des prélèvements en eau. } \\
\text { - Faible organisation pour la gestion des retenues } \\
\text { collinaires, de l'irrigation et de l'assainissement } \\
\text { - Problèmes liés à la gestion et à la gouvernance de } \\
\text { l'eau } \\
\text { - Faible coordination des organismes liés à la gestion } \\
\text { de l'eau. } \\
\text { - Faible capacités de gestion des cités urbaines et des } \\
\text { problèmes environnementaux liés à l'eau qui s'y } \\
\text { rapportent }\end{array}$ \\
\hline OPPORTUNITÉS (Opportunities) & MENACES (Threats) \\
\hline $\begin{array}{l}\text { - De mobilisation, de transfert et de gestion } \\
\text { des ressources en eau. } \\
\text { - Des possibilités de transferts intra et } \\
\text { interrégionaux permettant de valoriser au } \\
\text { mieux une ressource souterraine importante } \\
\text { (proximité des nappes sahariennes). } \\
\text { - Mise en œuvre de nouvelles Politiques } \\
\text { comme la mise en œuvre de l'Impératif } \\
\text { Haut Plateau permettant de mobiliser les } \\
\text { financements et de développer les capacités } \\
\text { de gestion de la ressource en eau et de } \\
\text { l'assainissement. } \\
\text { - Un potentiel de meilleure connaissance de } \\
\text { la ressource en eau par le lancement } \\
\text { d'études importantes. }\end{array}$ & $\begin{array}{l}\text { - Changement du climat si défaut d'adaptation } \\
\text { (systèmes de production, stockage de l'eau) } \\
\text { - Développement non maîtrisé de l'irrigation à partir } \\
\text { d'eau souterraine. } \\
\text { - Développement } \\
\text { consommatrices d'eau en lien avec l'Impératif Haut } \\
\text { Plateau. } \\
\text { - Dégradation de la qualité des nappes liée à la } \\
\text { surexploitation (salinité). } \\
\text { - Dégradation de la qualité des eaux des retenues à } \\
\text { cause de la mauvaise gestion de l'assainissement } \\
\text { - Envasement des retenues limitant leur durée de vie } \\
\text { - La faiblesse des capacités de gestion des risques } \\
\text { naturels liée à l'eau. }\end{array}$ \\
\hline
\end{tabular}

Source : auteurs

\section{La Mobilisation Des Ressources En Eau En Algérie}

Pour faire face au stress hydrique qui est émanant principalement du contexte climatique, au très bas potentiel pluviométrique, l'Etat algérien a investi dans la construction de barrages et la réalisation de forages ainsi que le recours aux ressources dites non conventionnelles (dessalement de l'eau de mer, épuration des eaux usées). Pour compenser les déficits, l'Algérie mène une politique active, conciliant le recours à différents types d'aménagement (barrages et transferts interbassins, eaux d'épuration pour la mise en valeur de nouvelles terres, dessalement, exploitation et transfert vers les Hauts Plateaux des eaux des nappes profondes du Sahara) ainsi qu'à de nouveaux instruments de gestion comme les schémas directeurs d'aménagement, les agences de bassin, la législation de l'eau et de l'environnement [26]. D'après Bouchedja [27], les potentialités et les perspectives de la mobilisation des ressources en eau en Algérie se résument comme suit :

- En $2011: 2,7$ milliards m3/an (63 barrages)

- En $2030: 4,3$ milliards m3/an (121 barrages)

- Développement de la réutilisation des eaux usées (estimé à 1,2 millions m3/an en 2015)

- Dessalement (2,3 millions m3/jour). 
Le recours au dessalement de l'eau de mer et à l'épuration des eaux usées ainsi que la mise en œuvre de la politique des réserves hydriques régionales stratégiques, vont permettre à notre pays d'augmenter ses capacités de mobilisation de la ressource, d'en stabiliser le schéma d'affectation et de pérenniser l'irrigation agricole au-delà de l'aléa climatique.

Pour contrer le stress hydrique, les surfaces irriguées ont doublé entre 1960 et 2005, ce qui a permis d'augmenter les rendements agricoles. Cependant, la ressource étant limitée au sud, la surexploitation de nombreuses nappes s'est accentuée, ainsi que la salinisation des eaux et des sols et parfois la pollution. En outre, les zones sensibles (pastorales et semi-arides) sont restées marginalisées.

\section{La Gestion Des Ressources En Eau En Algérie}

Le terme gestion est utilisé dans son sens le plus large et souligne la nécessite de ne pas seulement se focaliser sur le développement des ressources en eau, mais aussi de gérer consciencieusement le développement de l'eau qui assure un usage durable pour les générations futures. on a la gestion directe a ce mode l'état gère l'eau individuellement, et la gestion déléguée ici la gestion de l'eau se fait par des sociétés étrangères : société des eaux et de l'assainissement d'Alger (SEAAL), société de l'eau et de l'assainissement de Constantine (SEACO), société de l'eau et de l'assainissement d'Oran (SEOR), société des eaux et de l'assainissement d'el-Taref et d'Annaba (SEATA) pour la gestion de l'eau potable et l'assainissement dans les grandes villes du pays [28].

\section{Cadre de gestion des ressources en eau}

L'instrument juridique principal de gestion de l'eau est la loi n 83-17 du 16/07/1983. Cette loi a fixé l'ordre des priorités de l'utilisation de l'eau, l'assiette des redevances et la qualité de la ressource. Elle préconise tantôt des recommandations, tantôt elle impose des obligations ou prévoit des responsabilités pénales.

Entre l'Etat et les différents utilisateurs de l'eau, l'entreprise de gestion et de distribution détient un rôle d'intermédiaire. Elle assure trois sortes de services. Le premier est lié à la production de l'eau ; des stations de pompage et du matériel hydraulique sont utilisés pour cela. Le second est inhérent aux études de plans d'adduction et de développement du réseau au fur et à mesure que de nouveaux quartiers urbains se réalisent et le troisième concerne la distribution et les aspects financiers.

En Algérie, le caractère stratégique est affirmé à travers le schéma national d'aménagement du territoire et la mise en œuvre et d'une nouvelle politique de mobilisation et de gestion des ressources en eau.

Le ministère des ressources en eau est l'autorité centrale responsable de l'élaboration et de la mise en œuvre de la politique nationale de l'eau et dispose de relais déconcentrés au niveau local avec les directions des ressources en eau de wilaya.

En 2001, des réformes institutionnelles ont modifié en profondeur les établissements publics à compétence nationale qui sont sous la tutelle du ministère des ressources en eau :

- l'Agence nationale des ressources hydrauliques (ANRH) est chargée d'étudier et d'évaluer les ressources en eaux et en sols irrigables ;

- l'Agence nationale des barrages et transferts (ANBT) est responsable de mobiliser et de transférer les ressources en eau vers les lieux d'utilisation;

- l'Algérienne des eaux (ADE) a pour mission de gérer tout le processus d'alimentation en eau potable et industrielle y compris la mise en œuvre des programmes d'investissements ;

- la gestion et le développement des infrastructures d'assainissement urbain sont la prérogative de l'Office national de l'assainissement (ONA) ;

- l'Office national de l'irrigation et du drainage (ONID) est chargé de gérer les périmètres d'irrigation que l'Etat et les collectivités locales lui concèdent; dans ce cadre, l'Office a également pour tâche de mettre en œuvre des stratégies pour rationaliser l'usage de l'eau d'irrigation.

Sur le plan législatif, la gestion du secteur de l'eau en Algérie relève principalement de la loi relative à l'eau [29]. La promulgation de cette loi relative à l'eau qui définit des principes nouveaux de gestion intégrée et oblige à lutter contre les pertes et les gaspillages. Cette loi a pour objet de fixer les principes et les règles 
applicables pour l'utilisation, la gestion et le développement durable des ressources en eau en tant que bien de la collectivité nationale [30].

La loi sur l'eau de 2005, comme l'a résumé BENBLIDIA, consacre le droit d'accès à l'eau et à l'assainissement pour tous et définit les principes sur lesquels se fondent l'utilisation, la gestion et le développement durable des ressources en eau :

- le droit d'accès à l'eau et à l'assainissement pour tous

- le droit d'utilisation des ressources en eau pour tous dans les limites de l'intérêt général

- la planification de la répartition et des aménagements dans le cadre d'unités hydrographiques naturelles*

- la prise en compte des coûts réels des services d'approvisionnement en eau à usage domestique, agricole et industriel et des services d'assainissement

- la récupération des coûts d'intervention publique liés à la protection quantitative et qualitative des ressources en eau, à travers des systèmes de redevances d'économie d'eau et de protection de sa qualité

- la systématisation des pratiques d'économie et de valorisation de l'eau par des procédés et des équipements appropriés ainsi que le comptage des eaux produites et consommées ; pour lutter contre les pertes et le gaspillage

- la concertation et la participation de tous les acteurs.

Cette loi apporte certaines dispositions innovantes et importantes:

- l'obligation d'élaborer un Plan national de l'eau et la planification de la gestion locale dans le cadre des bassins hydrographiques,

- l'établissement de règles régissant les systèmes de tarification de l'eau usages appuyées sur les coûts réels des services d'approvisionnement,

- la possibilité de concession ou de délégation du service public de l'eau à des personnes morales de droit public ou privé [31].

\section{La Stratégies De La Politique Hydraulique En Algérie}

La politique algérienne de gestion de l'eau est davantage axée sur la mobilisation de nouvelles ressources que sur la recherche d'une meilleure utilisation des ressources déjà disponibles [32]. L'Algérie continue à faire face à des défis majeurs en matière de mobilisation de la ressource en eau et de lutte contre les pollutions, pour pouvoir faire face à une demande en hausse induite par la croissance démographique et le développement économique et social du pays. La mise en place d'une politique rigoureuse de gestion de la demande s'avère nécessaire.

Pour la valorisation des ressources hydriques, le développement de l'irrigation s'est inscrit dans une politique hydraulique qui s'est voulue cohérente et harmonieuse. Une planification intégrée et globale au niveau des bassins hydrauliques a accompagné l'extension de l'irrigation et l'effort de mobilisation des ressources en eau.

Cet effort de mobilisation des ressources en eau, est basé sur la gestion de l'offre, par un mécanisme de gouvernance pour assurer la sécurité hydraulique sur l'ensemble du territoire algérien.

\section{Agences de Bassins Hydrographiques}

Avant la création en Algérie des agences de bassins hydrographiques, la gestion des ressources en eau était de type centralisé, ce qui n'a pas permis de satisfaire les besoins en eau et ce, malgré des investissements importants et couteux. Cette centralisation des décisions a engendré d'énormes problèmes, qui ont été aggraves par l'insuffisance de la protection de la ressource, surtout en termes de quantité et de qualité. Parallèlement, d'autres contraintes ont apparu, notamment le manque d'arbitrage des conflits d'usages situés à l'intérieur des limites administratives. Ce mode de gestion était devenu anachronique, par rapport aux gestions modernes et participatives des ressources en eau par les différents acteurs au sein du bassin 
hydrographique [33]. Dans cette vision, une nouvelle politique de l'eau a été créée et adoptée en 1996 et a vu la création de cinq agences de bassins hydrographiques sur le territoire national. Le ministère des ressources en eau coordonne l'ensemble des activités liées à l'eau au niveau national, chaque wilaya à une direction de l'hydraulique. Il existe aussi des sociétés nationales telles ANB (Agence nationale des barrages), AGEP (Agence Générale de l'eau potable), ... des entreprises de wilaya et depuis 1996, existe un nouveau découpage par bassin hydrographique.

Cette nouvelle politique de l'eau a introduit la notion de gestion intégrée de l'eau dans son milieu naturel, qui est le bassin hydrographique et également les notions d'universalité et de concertation. Parmi les principaux axes de cette nouvelle politique de l'eau :

- L'eau est vulnérable et doit faire l'objet d'une protection quantitative et qualitative

- La gestion de l'eau doit être intégrée et de ce fait, mettre en place les instruments de gestion et les outils règlementaires.

Les décrets exécutifs n ${ }^{\circ}$ 96-279 à 283 portant création de cinq Agences de Bassin Hydrographique couvrant le territoire national et les décrets exécutifs n ${ }^{\circ}$ 96- 284 à 288 portant création de cinq comités de bassins.

Les cinq Agences de Bassin Hydrographique regroupent les 19 bassins versants du pays.

\section{Tableau 5 : Répartition de la superficie par régions hydrographique et bassins versants en Algérie}

\begin{tabular}{|c|c|c|}
\hline $\begin{array}{l}\text { Bassins } \\
\text { hydrographiques }\end{array}$ & $\begin{array}{l}\text { Superficie en } \\
\text { km2 }\end{array}$ & Bassins versants \\
\hline Oranie-Chott Chergui & 77169 & $\begin{array}{l}\text { - Côtiers oranais } \\
\text { - Macta } \\
\text { - Tafna } \\
\text { - Chott Chergui }\end{array}$ \\
\hline Chéliff-Zahrez & 56227 & $\begin{array}{l}\text { - Côtiers Dahra } \\
\text { - Chéliff } \\
\text { - Chott Zahrez }\end{array}$ \\
\hline Algérois-Hodna- Soummam & 47431 & $\begin{array}{l}\text { - Côtiers algérois } \\
\text { - Sébaou } \\
\text { - Isser } \\
\text { - Soummam } \\
\text { - Chott Hodna } \\
\end{array}$ \\
\hline $\begin{array}{l}\text { Constantinois-Seybousse- } \\
\text { Mellegue }\end{array}$ & 44348 & $\begin{array}{l}\text { - Côtiers constantinois } \\
\text { - Kébir Rhumel } \\
\text { - Medjerda Mellegue } \\
\text { - Seybousse } \\
\text { - Hauts Plateaux } \\
\text { - Constantinois } \\
\end{array}$ \\
\hline Sahara & 2018054 & - Sahara \\
\hline
\end{tabular}

Source : Ministère des Ressources en Eau

Les ressources en eau superficielle renouvelables internes totalisent environ $9.8 \mathrm{~km} 3 / \mathrm{an}$ pour l'ensemble du pays dont le Sahara, bassin le plus important par la surface, ne renferme que $0.6 \mathrm{~km} 3$. Les ressources en eau souterraine renouvelables contenues dans les nappes du nord du pays sont estimées à près de $1.5 \mathrm{~km} 3 / \mathrm{an}$. Ces nappes sont alimentées essentiellement par les précipitations dont la répartition demeure irrégulière à la fois dans le temps et dans l'espace.

Le sud du pays se caractérise par l'existence de ressources en eau souterraines très importantes provenant des nappes du continental intercalaire et du complexe terminal.

Dans ce contexte, un scénario volontariste repose sur une volonté politique et publique : 
- La volonté politique se présente par la réduction des pertes à partir de renouvellement des conduites et par la réutilisation des eaux usées et la sensibilisation et de lancer des formations pour faire l'économie ...etc. développement de nouveaux système d'information, établissement les plans d'action de gestion des ressources en eau, évaluation et valorisation des ressources en eau .

- La volonté publique par le soutien de la politique volontariste par le gouvernement et les établissements sous tutelles (AGIRE, ANRH, ABH, ANBT, ONID) à partir de faire l'économie d'utilisation de l'eau [34].

\section{Missions des Agences de Bassin Hydrographique}

Les agences de bassin sont créées avec mise en place progressive de dispositifs et d'outils réglementaires pour la gestion quantitative et qualitative de l'eau. Le statut de ces agences de bassins hydrographiques est : établissement public à caractère industriel et commercial (EPIC).

Le principe de la concertation avec les différents partenaires (agriculture, industrie, associations d'usagers, collectivités locales, universités, ...) est retenu. Le code des eaux a été modifié et permet notamment la concession d'installation d'eau potable et/ou d'assainissement à des opérateurs privés. La concertation est assurée par un comité de bassin composé à parts égales, de représentants de l'administration, des élus locaux, et des usagers. Il peut discuter de toutes les questions d'eau au sein du bassin hydrographique. Le développement d'une prise de conscience par l'ensemble des acteurs de l'eau est un objectif permanent afin de préserver la ressource en eau [35]. Ces agences sont chargées :

- De développer le système d'information sur l'eau à travers l'établissement et l'actualisation de bases de données et d'outils d'informations géographique.

- D'établir les plans de gestion des ressources en eau superficielles et souterraines et élaborer des outils d'aide à la décision en la matière.

- De gérer le système de redevance institué au titre de l'utilisation du domaine public Hydraulique naturel, qui lui est confié.

- De gérer le système d'aides financières aux actions visant l'économie de l'eau et la préservation de sa qualité, qui lui est confié.

- De mettre en œuvre et promouvoir tous projets et actions visant à rationaliser l'utilisation des ressources en eau et à prévenir leur pollution.

- Assurer les suggestions de service public mises à sa charge par l'état dans le domaine de la gestion de l'eau.

\section{Missions des comités de bassin}

Les Comités de bassin ont pour mission de débattre et de formuler un avis sur toutes les questions liées à l'échelle de chaque bassin hydrographique et notamment sur :

- l'opportunité des travaux et d'aménagements hydrauliques envisagés dans les bassins, les différents de tous types liés à l'eau, pouvant survenir entre les collectivités locales.

- la répartition de la ressource en eau mobilisée entre les différents utilisateurs potentiels

- les actions à envisager pour les protections quantitatives et qualitatives de la ressource en eau

- les programmes d'interventions des agences de chaque bassin hydrographique.

Les missions, la composition et le fonctionnement relatifs aux cinq comités de bassins hydrographiques ont été fixés par le décret exécutif $\mathrm{n}^{\circ} 10.24$ du 12 janvier 2010 définissant le cadre de concertation en matière de gestion intégrée des ressources en eau.

\section{La redevance prélèvement : Outil pour une incitation aux économies d'eau}

En 2005 et 2006, les activités ont découlé de la Loi de Finances algérienne de juillet 2005 qui confie aux Agences de Bassin Hydrographique (ABH) la perception de redevances pour l'usage direct des ressources en eau du "Domaine Public Hydraulique". Il s'agit d'inciter les utilisateurs d'eau à mieux gérer l'eau 
prélevée dans le milieu naturel, en leur faisant supporter un coût d'usage à travers cette redevance prélèvement.

Cette action est déterminante pour les Agences de Bassin Hydrographique, car une partie de la redevance collectée pourra être utilisée pour financer des actions, telles que la planification, le développement de la gestion intégrée des ressources en eau, la sensibilisation des usagers aux économies d'eau, mais aussi le financement de mesures pour favoriser le recyclage, la lutte contre le gaspillage, etc. Cette redevance est versée au compte d'affectation spéciale $\mathrm{n}^{\circ}$ 302-086 intitulé «Fonds National de Gestion intégrée des Ressources en Eau".

Les produits de ces redevances sont destinés à assurer la participation de l'usager aux programmes de protection quantitative et d'économie des ressources en eaux (Article 173 de l'ordonnance $\mathrm{n}^{\circ}$ 95-27 du 3012-1995).

Afin d'améliorer la redevance prélèvement, adoptée depuis la création des Agences de Bassin Hydrographique (ABH) algériennes en 1996, un appui institutionnel et juridique a été apporté par l'Office International de l'Eau et l'Agence de l'Eau Seine-Normandie au début 2008. La redevance prélèvement est considérée comme outil économique d'incitation à une meilleure gestion des ressources en eau et à la préservation des ressources en eau en Algérie.

Les déficits en eau exigent la mise en place d'une politique rigoureuse de gestion de la demande, fondée sur des mécanismes financiers et réglementaires, ainsi que sur la production d'une information complète concernant la situation hydrologique.

\section{La Gestion Intégrée Des Ressources En Eau (Gire)}

La gestion est entendue dans sa définition la plus primaire. Il souligne que nous ne devons pas seulement nous focaliser sur l'exploitation des ressources en eau, mais plutôt gérer consciencieusement l'exploitation des réserves d'eau, de façon à assurer à long terme un usage soutenu continu pour les générations futures.

La Gestion Intégrée des Ressources en Eau, correspond à l'IWRM -Integrated Water Resources Management. L'IWRM a notamment été promu par le Partenariat Mondial de l'eau (GWP), en 2000. Ce programme, associé à une méthode, est axé sur la gestion des bassins versants, considérés à l'échelle du fleuve. L'IWRM est « un processus favorisant le développement et la gestion coordonnés des ressources en eau, du sol et des ressources associées, permettant de maximiser les bénéfices économiques et sociaux, de façon équitable sans compromettre la pérennité des écosystèmes vitaux » [36]. La GIRE est, surtout, une philosophie. Comme telle, elle offre un cadre conceptuel de gouverne dans le but d'une gestion et une mise en valeur, durable des ressources en eau. Elle cherche également à introduire un élément de démocratie décentralisée dans la façon dont l'eau est gérée, avec une insistance sur la participation des parties prenantes et la prise de décision au niveau inférieur le plus approprié.

En Algérie, la loi de 2005 a réformé l'ensemble du dispositif institutionnel de gestion intégrée des ressources en eau (GIRE) qui avait été initialement mis en place en 1996 en s'inscrivant dans l'approche adoptée par la déclaration de la conférence internationale sur l'eau et l'environnement de Dublin (janvier 1992) et la déclaration de la conférence de Rio sur l'environnement et le développement appelée Sommet de la Terre (juin 1992).

Le dispositif institutionnel de la GIRE a été récemment réorganisé avec la création de l'agence nationale de gestion intégrée des ressources en eau «AGIRE» par décret exécutif du 30 juillet 2011. Cette réorganisation vise à renforcer davantage les capacités d'intervention du secteur dans ce domaine notamment à travers la coordination des activités et une répartition mieux équilibrée des ressources financières mobilisées par le fonds national de gestion intégrée des ressources en eau (FNGIRE).

Dans ce nouveau schéma organisationnel, les ABH sont rattachées à l'AGIRE en tant que démembrements territoriaux où continuera à s'exercer la concertation au sein des comités de bassins.

La gestion intégrée des ressources est la prise en charge de la gestion des ressources souterraines par le suivi et le contrôle des prélèvements, la mise en place d'une gestion collective à l'échelle locale, la mise en place des droits d'usage et des redevances d'usages. 
La satisfaction continue de la demande en eau et spécialement durant les périodes de sécheresse, nécessite une infrastructure propre à satisfaire la demande de ressources en eau alternatives. La réalisation d'infrastructures de transfert et de connexions entre systèmes hydrauliques constitue la piste la plus plausible pour assurer la sécurité hydraulique en Algérie.

L'engagement de la politique de l'eau sur la voie de la gestion de la demande s'opère d'une manière assez timide. Cette stratégie doit être renforcée d'une manière progressive afin de parvenir à une véritable gestion de la demande, seule voie pour relever les défis qui se posent au développement et à la gestion des ressources en eau. La connaissance de cette politique conduit à consolider l'approche participative dans la gestion des ressources en eau à travers le développement des associations des usagers de l'eau, concourant à l'intérêt collectif d'une utilisation efficiente et conservatrice de ces ressources. Pour assurer notre sécurité en eau, il serait souhaitable de mettre en œuvre de nouvelles pratiques de gestion économes et durables du patrimoine hydrique et la mise en place « de politiques de bonne gouvernance des ressources en eau ».

\section{Conclusion}

L'eau est au cœur des enjeux de demain, il pourrait être un générateur des conflits [37] (internes ou externes) surtout dans les pays vulnérable aux changements climatiques comme l'Algérie. En effet, l'Algérie pourrait souffrir de pénuries du fait du changement climatique, qui affecte les quantités d'eau disponibles, et de l'augmentation de la demande en eau. La politique de l'eau à mener aux cours des années à venir est à focaliser sur une gestion plus efficace d'une ressource de plus en plus rare. Toute en valorisant l'existant, elle devra privilégier la gestion de la demande plutôt que celle de l'offre, qui a atteint ses limites. La réussite d'une gestion intégrée de l'eau suppose une réelle implication des élus et des usagers qui sont appelés à contribuer activement à la prise de conscience sur les enjeux de l'eau au moment où les impacts de changements climatiques sont de plus en plus significatifs dans notre pays. En effet, ces changements climatiques ont une incidence à la fois sur les potentialités en eau mais aussi sur leurs fluctuations temporelles. La politique de gestion des ressources en eau doit être basée sur des axes stratégiques comme l'amélioration de l'existant, la mobilisation et la préservation des ressources en eau pour assurer la satisfaction des besoins en eau et sa protection quantitative et qualitative dans le cadre d'une bonne gouvernance de l'eau. La planification des ressources en eau doit prendre en compte les stratégies de mobilisation des ressources conventionnelles (construction de nouveaux barrages et développement les systèmes de transfert, stratégies d'exploitation des ressources souterraines) ainsi que les stratégies de mobilisation des ressources non conventionnelles (le dessalement de l'eau de Mer, réhabilitation et extension des systèmes d'AEP, d'assainissement, etc.).

\section{References}

[1.] M. MOZAS, A. GHOSN, “État des lieux du secteur de l'eau en Algérie”. L'Institut de prospective économique du monde méditerranéen, 2013, IPEMED. Site web : www.ipemed.coop,

[2.] F. SENHADJI F., M. SENOUCI, "Les changements climatiques dans les pays du Maghreb" in MED énergie. Revue méditerranéenne de l'énergie. Numéro hors-série. 2003.

[3.] M. MILANO, «Les impacts prévisibles du changement climatique sur les ressources en eau de quatre grands bassins versants Méditerranéens », Plan Bleu, Sophia Antipolis janvier 2010. Plan bleu.com

[4.] M. MILANO M. (2010) : «Les impacts prévisibles du changement climatique sur les ressources en eau de quatre grands bassins versants Méditerranéens », Plan Bleu, Sophia Antipolis janvier 2010. Plan bleu.com

[5.] S. SIMONET, “Adaptation au changement climatique dans le secteur de l'eau en méditerranée : situation et perspectives." Les Cahiers du Plan Bleu 10, Septembre 2011. Site web : www.planbleu.org

[6.] C. BULLE, "Changements climatiques et usages en eau dans le bassin méditerranéen: impacts et adaptations". Office International de l'Eau (CNIDE), ENGREF Centre de Montpellier. Février 2008.

[7.] M. MEDDI, A. TALIA et C. MARTIN, « Évolution récente des conditions climatiques et des écoulements sur le bassin versant de la Macta (Nord-Ouest de l'Algérie) », Physio-Géo [En ligne], Volume 3, 2009, mis en ligne le 01 janvier 2009, Consulté le 20 février 2015. URL : http://physiogeo.revues.org/686 ; DOI : 10.4000/physio-geo.686 
[8.] O. BENALIA, «Modélisation de la demande en eau dans une région aride. Cas de la Wilaya de Djelfa », Revue « Nature \& Technologie ». $\mathrm{N}^{\circ}$ 06/Janvier 2012 http://www.univchlef.dz/RevueNatec/art_06_12.pdf.

[9.] G. BENOIT, A. COMEAU, « Le bassin méditerranéen va connaître une crise de l'eau douce » [en ligne]. Rome, Réseau International des Organismes de Bassin, 2007.

www.inbo-news.org/euroriob/rome/comms/RIOB_Roma_2007_OIEAU_crise.pdf>

[10.] C. BULLET, "Changements climatiques et usages en eau dans le bassin méditerranéen : impacts et adaptations". Office International de l'Eau (CNIDE), ENGREF Centre de Montpellier. Février 2008.

[11.] S. DJAFFAR, A. KETTAB, "La gestion de l'eau en Algérie : quelles politiques, quelles stratégies, quels avenirs ?", Algerian J. Env. Sc. Technology, 4:1 (2018) 641-648

[12.] B. REMINI, "La problématique de l'eau en Algérie du nord". Larhyss Journal, ISSN 1112-3680, $\mathrm{n}^{\circ}$ 08, Juin 2010, pp. 27-46.

[13.] B. REMINI, “La problématique de l'eau en Algérie du nord”. Larhyss Journal, ISSN 1112-3680, $\mathrm{n}^{\circ}$ 08, Juin 2010, pp. 27-46.

[14.] KETTAB, “L'eau au 3ème millénaire : enjeux, stratégies, politiques, défis, vision”, Conférence plénière à l'occasion de la journée mondiale de l'eau, Ecole Nationale Polytechnique, Alger, Mars 2000.

[15.] M. COTE, “L’Algérie : espace et société”. Paris, Armand Colin, 1996. p.p. 30-41.

[16.] M. COTE, “L’Algérie : espace et société”. Paris, Armand Colin, 1996. p.p. 30-41.

[17.] P. LABORDE, A. MEBARKI, "Bases d'information et cartographie des bilans hydriques de l'Algérie du Nord", 1er Colloque International sur la Gestion Intégrée des Ressources en Eau GIRE, Batna, Algérie, 10-11 novembre 2009, Proceedings, 72-75.

[18.] M. COTE, "L’Algérie : espace et société". Paris, Armand Colin, 1996. p.p. 30-41

[19.] MOUSTIRI, "Stratégie et indicateurs du secteur de l'eau en Algérie", le Caire 21-22 novembre 2011.

[20.] L. BROURI, "Impacts des changements climatiques sur la gestion durable des ressources pastorales et des parcours dans les zones arides et semi arides de l'Algérie" "L'effet du Changement Climatique sur l'élevage et la gestion durable des parcours dans les zones arides et semi-arides du Maghreb »Université KASDI MERBAH - Ouargla- Algérie, du 21 au 24 Novembre 2011. http://manifest.univ-ouargla.dz/

[21.] M. MILANO, «Les impacts prévisibles du changement climatique sur les ressources en eau de quatre grands bassins versants Méditerranéens », Plan Bleu, Sophia Antipolis janvier 2010. Plan bleu.com

[22.] M. MILANO, « Les impacts prévisibles du changement climatique sur les ressources en eau de quatre grands bassins versants Méditerranéens », Plan Bleu, Sophia Antipolis janvier 2010. Plan bleu.com

[23.] M. BENBLIDIA, G. THIVET, « Gestion des ressources en eau : les limites d'une politique de l'offre ». Plan Bleu. Les Notes d'analyse du CIHE AM. N 58 - Mai 2010 www.ciheam.org

[24.] M. BENBLIDIA, G. THIVET, « Gestion des ressources en eau : les limites d'une politique de l'offre ». Plan Bleu. Les Notes d'analyse du CIHE AM. N 58 - Mai 2010 www.ciheam.org

[25.] Ministère des Ressources en Eau, 2012

[26.] MEBARKI, "La région du Maghreb face à la rareté de l'eau. L'exemple du défi algérien : mobilisation et gestion durable des ressources". Second International Conference: Climate, Sustainability and Development in semi-arid regions August 16-20, 2010, Fortaleza - Ceará, Brazil.

[27.] BOUCHEDJA, « La politique nationale de l'eau en Algérie » In Euro-RIOB 2012, 10ème Conférence Internationale, Istanbul - Turquie - 17 au 19 Octobre 2012.

[28.] S. DJAFFAR, A. KETTAB, "La gestion de l'eau en Algérie : quelles politiques, quelles stratégies, quels avenirs?”, Algerian J. Env. Sc. Technology, 4:1 (2018) 641-648

[29.] LOI RELATIVE A L'EAU DU 4 AOUT 2005 publiée dans le Journal officiel de la république algérienne démocratique et populaire JORA n ${ }^{\circ} 60 \mathrm{du} 4$ septembre 2005.

[30.] JOURNAL OFFICIEL N 60 DU 4 septembre 2005. 
[31.] M. BENBLIDIA, « Amélioration de l'efficience de l'utilisation de l'eau et approche économique de la gestion de la demande en eau » : rapport national de l'Algérie, étude réalisée pour le plan bleu. Sophia Antipolis (France), juin 2011.

[32.] M. BENBLIDIA, G. THIVET, « Gestion des ressources en eau : les limites d'une politique de l'offre ». Plan Bleu. Les Notes d'analyse du CIHE AM. N 58 - Mai 2010 www.ciheam.org

[33.] KHADRAOUI, "Présentation des enjeux du Bassin de l'Agence de bassin Hydrographique « Sahara 》 en matière de gestion intégrée des Ressources en eau - Cas du bassin Sahara septentrional". In Colloque tenu au Centre Universitaire Abbès LAGHROUR de KHENCHELA, les 6 et 7 décembre 2011 intitulé « développement durable des territoires et zones arides ou semiarides dans le bassin méditerranéen ».

[34.] S. DJAFFAR, A. KETTAB, “La gestion de l'eau en Algérie : quelles politiques, quelles stratégies, quels avenirs ?", Algerian J. Env. Sc. Technology, 4:1 (2018) 641-648A. KETTAB, "Les ressources en eau en Algérie : stratégies, enjeux et vision”. Desalination 136 (2001) 25-33

[35.] LES NOUVELLES $\mathrm{N}^{\circ} 19$ - décembre (2008) : Algérie : Appui institutionnel sur les redevances des Agences de Bassin (en ligne) http://www.oieau.fr/oieau/notre-actualite-et-avancementde/article/algerie-appui-institutionnel-sur?lang=fr

[36.] GLOBAL WATER PARTNERSHIP (GWP), "Manuel de Gestion Intégrée des Ressources en Eau par Bassin”. Publié en 2009 par le Partenariat mondial de l'eau (GWP) et le Réseau international des organismes de bassin (RIOB), http:// www.gwpforum.org http://www.inbo-news.org

[37.] S. DJAFFAR, A. KETTAB, "La gestion de l'eau en Algérie : quelles politiques, quelles stratégies, quels avenirs ?”, Algerian J. Env. Sc. Technology, 4:1 (2018) 641-648. 